\title{
A forgotten nosocomial bacterium (Chryseobacterium meningosepticum)
}

\author{
O Kítchin, MR Lekalakala, JA Joshi, A Meyer, R Masekela, SM Risenga, T Moodley, TJ Avenant, RJ Green
}

0 Kitchin, MR Lekalakala, JA Joshi, A Meyer, R Masekela, SM Risenga, T Moodley, TJ Avenant, RJ Green, Department of Paediatrics and Child Health, University of Pretoria.

South Afr J Epidemiol Infect 2009;24(4):38

We report on two cases referred to our paediatric intensive care unit(PICU) from a paediatric surgery ward with sepsis due to Chryseobacterium meningosepticum.

Patient A is a 6-day-old female with a tracheo-oesophageal fistula admitted to the PICU with apnoea requiring artificial respiratory support. She had septic shock, with a disseminated intravascular coagulopathy and had clinical and radiological pneumonia. She was resuscitated, recovered and was successfully extubated within seven days of admission to PICU. Chryseobacterium meningosepticum was recovered from blood and cerebrospinal fluid cultures.

Patient $B$ is a 3-week-old female with a gastroschisis admitted to the PICU with septicaemia. She had a temperature of $38^{\circ} \mathrm{C}$ and required artificial respiratory support. She had been in the paediatric surgical ward for six days. She recovered well and was extubated within 48 hours of admission to the PICU. Fourteen hours later she developed apnoea necessitating re-ventilation. A full fontanel was noted. A lumbar puncture specimen grew Chryseobacterium meningo-septicum, also grown from the blood culture and endotracheal aspirate taken on admission. Empiric antibiotics used for both patients were amikacin and meropenem but were changed to ciprofloxacillin on obtaining the sensitivities reported 72 hours after specimens were obtained.

Susceptibility patterns of both isolates are shown in Table 1.

\section{Discussion}

Chryseobacterium meningosepticum is a Gram-negative, oxidasepositive, indole-positive, yellow pigmented nonfermenting bacillus. It appears smooth and fairly large (1-2 $\mathrm{mm}$ in diameter) on culture plates. The yellow pigment is due to the production of the water insoluble pigment, flexirubin. ${ }^{1}$ Chryseobacterium spp are inherently resistant to many antimicrobials commonly used to treat Gram-negative infections but often susceptible to agents used for Gram-positive bacteria, namely rifampicin, clindamycin, erythromycin, levofloxacin, trimethoprimsulfamethoxazole and vancomycin..$^{1,2}$

Table 1: Results of antibiotic susceptibility testing from blood and cerebrospinal fluid cultures for patient A and B, using Vitek-2 identification and susceptibility testing method

\begin{tabular}{|l|c|c|c|c|}
\hline Antibiotic & $\begin{array}{c}\text { Blood } \\
\text { culture A }\end{array}$ & $\begin{array}{c}\text { Blood } \\
\text { culture B }\end{array}$ & $\begin{array}{c}\text { Cerebrospinal } \\
\text { fluid A }\end{array}$ & $\begin{array}{c}\text { Cerebrospinal } \\
\text { fluid B }\end{array}$ \\
\hline Ciprofloxacin & S & S & S & S \\
\hline Vancomycin & S & R & R & R \\
\hline Amikacin & S & R & S & R \\
\hline Cotrimoxazole & S & R & S & R \\
\hline Rifampicin & S & R & S & R \\
\hline Streptomycin & R & R & R & R \\
\hline
\end{tabular}

$\mathrm{S}=$ Susceptible R=Resistant
Although early investigators recommended vancomycin for treating serious infections, subsequent studies have shown greater in vitro activity of minocycline, rifampicin, trimethoprim-sulfamethoxazole and the quinolones. However, combination therapy remains most recommended. ${ }^{2}$

In our laboratory we use the automated system Vitek-2 for identification and susceptibility testing of all Gram negative bacilli.

Most reports of Chryseobacterium meningosepticum are as outbreaks in neonatal units, usually premature infants in the first two weeks of life. ${ }^{3}$ Neonatal meningitis is the most fatal form of disease with mortality of up to $57 \%$ and post infection sequele i.e. hydrocephalus and developmental delay are common. ${ }^{4}$

In our unit, the hospital infection control team identified that the possible source of infection was related to a break in infection control practices. The unit of origin scored $83.5 \%$ on an environmental audit and $72.5 \%$ on a hand hygiene audit done when the two patients were transferred to the PICU. There was a 50\% compliance with a hand scrub (chlohexidine-based solution) and hand washing between patients. This was monitored daily with subsequent improvement. There have been no further outbreaks.

In a previous report, the most effective antimicrobials were the quinolones followed by rifampicin, with vancomycin demonstrating poor potency and resistance demonstrated to the aminoglycosides and carbapenems. ${ }^{4,5}$ Our patients demonstrated mixed antimicrobial patterns of susceptibility on both the blood and cerebrospinal fluid cultures. The organisms from both patients retained ciprofloxacin susceptibility. Even though rifampicin is an effective antimicrobial, in our environment we elected not to use it because tuberculosis is endemic and sensitivity was demonstrated in only one patient. Chryseobacterium meningosepticum meningitis can be fatal. However our patients' survived. We attribute survival to use of ciprofloxacin following early organism identification. The long term adverse effects of ciprofloxacin have been debated in the literature with Van den 0ever ${ }^{6}$ and colleagues reporting no adverse effect in a three-year follow-up of neonates who had infections resistant to other antimicrobials.

\section{References}

Murry P, Jo Baron E, Jorgensen J, Landry ML, Pfaller MA, eds. Oxidase positive, indole positive yellow pigmented nonfermenters. In: Manual of clinical microbiology, 9thed. Washington; 2007: 791 Di Pentima MC, Mason jr E0, Kaplan SL. In vitro antibiotics synergy against flavobacterium meningosepticum: implications for therapeutic options. Clin Infect Dis 1998; 23: 1169-1179 Gungor S, Ozen M, Akinci A, et al. A Chryseobacterium meningosepticum outbreak in a neonatal ward. Infect Control Hosp Epidemiol 2003; 24: 613-617

4. Kirby JT, Sader HS, Walsh TR, et al. Antimicrobial susceptibility and epidemiology of a worldwide collection of Chryseobacterium spp.: Report from the SENTRY Antimicrobial Surveillance Program (1997-2001). J Clin Microbiol 2004; 42: 445-448

5. Gales AC, Jones RN, Andrade SS, et al. Antimicrobial susceptibility patterns of unusual nonfermentative gram-negative bacilli isolated from Latin America: report from the SENTRY Antimicrobial Surveillance Program (1997-2002). Mem Inst Oswaldo Cruz 2005; 100: 671-677

6. Van den Oever HL, Versteegh FG, Thewessen EA, et al. Ciprofloxacin in preterm neonates: case report and review of the literature. Eur J Pediatr 1998; 157: 843-845 